\title{
Inhaltsverzeichnifs des ein und dreifsigsten Bandes, nach den Gegenständen.
}

\author{
I. Reine Mathematik.
}

Nr. der

A bhandlum:

1. A n a l y s i s.

1. Entwicklung eines symmetrischen Ausdrucks für den Grad einer durch

Heft Seite Elimination hervorgehenden Gleichung. Von Herrn Fcrd. Mirdiny, Prof. an der Universität zu Dorpat. (Gelesen in der Silzung der Petersburger Akademie der Wissenschaften am $\frac{24 \text {. Nov. }}{6 . \text { Dectrr. }} 1843$ und aus dem Bulletin übersetzt.)

2. Nuove applicazioni del Calcolo Integrale relative alla quadratura delle Superficie curve, e cubatura de solidi. Memoria dal Sign. D. Barnaba Tortolini, Professore di Matematiche trascendenti all'Università di Roma.

1. 12

๙. Summation der Reihe $\frac{1}{(b+a)^{1+\varphi}}+\frac{1}{(b+2 a)^{1+\varphi}}+\frac{1}{(b+3 a)^{1+\varrho}}+\cdots$ für $\varrho=0$. Von Herrn Dr. IIeine zu Bonn. . . . . . . . . . . . . .

10. Note sur la division abrégée en arithmélique. Par l'éditeur. . . . .

13. Über die Anzahl und die Forın der Bedingungsgleichungen, unter welchen eine gewöhnlıche Differentialgleichung zwischen zwei Variabeln $n$ ter Ordnung und von der Form

$$
V=y_{n} \varphi\left(x, y, y_{1}, y_{2}, \ldots y_{n-1}\right)+\psi\left(x, y, y_{1}, y_{2}, \ldots y_{n-1}\right)=0
$$

das unmittelbare Differentiations-Ergebnifs einer nach dẹr allgemeinen Constante aufgelöseten analogen Differentialglelchung $(n-1)$ ter Ordnung ist. Von Herrn Prof. Raabe zu: Zürich. . . . . . . . . . . . . . .

16. Zwei Beweise für die Existenz der Wurzeln der höhern algebraischen Gleịchungen. Von Herrn J. C. Ullherr, Professor an der polytechnischen Schule zu Nürnberg. . . . . . . . . . . . . . . . . . . .

17. Über die Summirung der beiden Reihen

$$
\begin{array}{ll}
\text { (a) } \gamma_{0}-n_{1} \gamma_{1}+n_{2} \gamma_{2}-\text { elc. }+(-1)^{n} \gamma_{n}, \\
\text { (b) } \gamma_{0}+n_{1} \gamma_{1}+n_{2} \gamma_{2}+\text { etc. }+\gamma_{n},
\end{array}
$$

in welchen die Gröfsen $\gamma$ willkürlich und die Coefficienten Binomialcoêfficienten des ganzen Exponenten $n$ sind, mittels höherer Differenzen und Summen. Von dem Herrn Gymnasiallehrer F. Arudt zu Stralsund. . .

18. Nova solutio problematis determinandi mulitudinem numerorum, qui ad numerum aliquem sint primi eoque minores. Anctore Eriderico Arndt, Sundiae.

19. Entwickelung der Summe der uten Potenzen der vatürlichen Zahlen nach den Potenzen des Index mittels des Taylor'schen Lehrsatzes. Von dem Herrn Gymnasiallehrer Arudt zu Stralsund. . . . . . . . . . . .

20. C̈ber die Bernoull'sche Methode, summirbare Reihen zu finden. Von dem Herrn Gymnasiallehrer Arrult zu Stralsund. . . . . . . . . . . . III. 253

21. Nova methodus determinandi multitudinem radicum congruentiae $x^{t} \equiv \mathbf{1}(\bmod . M)$ aliaque ad hanc materiam spectantia. Auctore Friderico Arndt, Sundiae. . . III. 259

22. Grundzũge einer allgemeinen Theorie der höhern Congruenzen, deren Modul eine reelle Primzahl ist. Von Herrn Oberlehrer Schonemann am Gymnasio zu Brandenburg a. d. H. . . . . . . . . . . . . . . . . . 
23. Demonstratio duorum theorematum Gaussianis his generaliorum:

I. Productum ex omnibus radicibus primitivis moduli imparis p unitate sec. $\mathrm{p}$ congruum est, excepto casu, in quo $\mathrm{p}=3$.

II. Summa ommium radicum primitivarum moduli primi imparis p est $\equiv 0$, quando $\mathrm{p}-1$ per quadratum aliquod divisibilis est; quando vero per nullum quadratum divisibilis, summa est $\equiv \pm 1$, prout multitudo factorum ipsius $\mathrm{p}-1$ primorum est par

Auctore Friderico Arndt, Sundiae. ant impar

24. Demonstratio nova theorematis Wilsoniani a summo Gauss hoc modo generalius enunciati:

„Productum omnium numerorum ad numerum quemcunque $\mathbf{M}$ pri„morum eoque inferiorum unitati negativae aut positivae sec. $M$ con,gruum est; et quidem neyative sumenda est unitas, quando $\boldsymbol{M}$ „potestas numeri primi imparis vel ejus duplum, vel denique 4, po„sitive autem in omnibus casibus reliquis."

Auctore Friderico Arndt, Sundiae.

25. Disquisitiones de residuis cujusvis ordinis. Auct. Friderico Arndt, Sundiac.

26. Bemerkungen über die Verwandlung der irrationalen Quadratwurzel in einen Kettenbruch. Von Herrn Dr. Arvdt zu Stralsund.

2. G e.o m e t ri e.

3. Auflösungen und Beweıse einer Reihe von Aufgaben und Lehrsätzen der ebenen Geometrie. Von Herrn A. Jacobi zu Breslau, Premier-Lieutenant a. D.

6. Beschlufs dieser Abhandlung.

II. 93

4. Einige geometrische Aufgaben. Von Herrn Prof. Lehmus in Berlin.

I. 85

5. Geometrische Lehrsätze und Aufgaben. Von Herrn Prof. J. Steiner in Berlin.

I. 90

7. Grundzüge zu einer rein geometrischen Theorie der Curven, mit Anwendung einer rein geometrischen Analyse. Von Herrn Dr. Herrman Gra/smann, Lehrer der Mathematik zu Stettin. . . . . . . . . . . . . . .

12. Beweis eines geometrischen Satzes. Von dem Premier-Lieutenant a. D. Herrn A. Jacobi. . . . . . . . . . . . . . . . . . . . .

4. Sur quelques théorèmes de la géométrie de position. Par Mr. A. Cayley de Cambridge.

15. Problème de géométrie analytique. Par' Mr. Cayley de Cambridge.

III. 213

III. 227

\section{Angew and te Mathematik.}

9. Recherches sur les surfaces isothermes et sur l'attraction des ellipsoïdes par M. Ch. Despeyrous à Paris, Docteur es-sciences. .

11. Elementare Herleitung des Newtonschen Gesetzes aus den Keplerschen Gesetzen der Planetenbewegung. Von Herrn A. F. Möbius, Professor in Leipzig.

Fac simile einer Handschrift von Gal. Galilaci. . . . . . . . . . . I

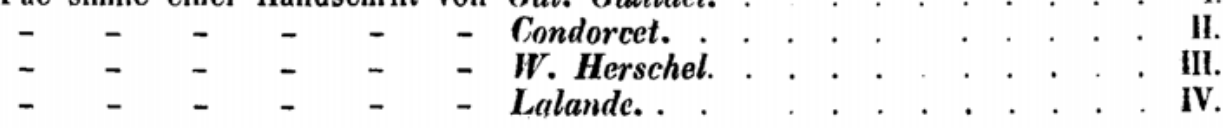

